\title{
Surgical management of symptomatic cardiac fibromas in children
}

\author{
Ben Davies, MRCS(Eng), ${ }^{a}$ Guido Oppido, MD, and Christian P. Brizard, MD, MS, a,b Melbourne, Australia
}

S Supplemental material is available online.

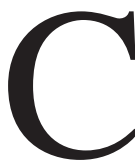
ardiac fibromas have the second highest incidence of primary cardiac tumors in children after rhabdomyomas but are the most commonly resected. ${ }^{1}$ In contrast to rhabdomyomas, fibromas are usually large, solitary, intramural tumors with a predilection for the left ventricular free wall or interventricular septum ${ }^{2}$ and tend not to regress spontaneously. They may be asymptomatic or present with arrhythmias or cardiac failure due to chamber obliteration and valvular obstruction. ${ }^{1}$ We describe 2 cases in which resection and reconstruction of giant intramural cardiac fibromas involving the atrioventricular (AV) groove was possible without resorting to mechanical circulatory support or transplantation.

\section{Clinical Summary}

PATIENT 1. A 3-day-old boy $(3.5 \mathrm{~kg})$ presented with cyanosis and cardiac failure shortly after birth. Preoperative evaluation with echocardiography and computed tomography demonstrated a 7-cm $\mathrm{m}$ ass involving the anterior aspect of the right atrium and right ventricular (RV) outflow free wall, resulting in compromise of the $\mathrm{RV}$ cavity and intermittent obstruction of both caval inflow and RV outflow.

At operation, the hourglass-shaped tumor was found to have no distinct margin and had infiltrated the AV groove, incorporating the right coronary artery. The mass was resected en bloc, removing $\mathrm{RV}$ free wall, a portion of the anterior tricuspid annulus, a portion of right atrium, and the second part of the right coronary artery. The resulting defect was repaired with a double layer of glutaraldehyde-treated autologous pericardium reinforced with sutures and fibrin glue. Continuity of the tricuspid valve annulus was restored by suturing together RV and right atrial patches incorporating the exposed hinge of the anterior leaflet (Figures 1; E1). Bypass was weaned easily with minimal inotropic support and a nonischemic electrocardiogram. The chest was left open electively and later closed with the patient being extubated after 4 days.

From the Cardiac Surgical Unit, Royal Children's Hospital, a and the Department of Paediatrics, University of Melbourne, ${ }^{\mathrm{b}}$ Melbourne, Australia.

Received for publication Sept 13, 2006; accepted for publication Sept 19, 2006.

Address for reprints: Ben Davies, MRCS(Eng), Cardiac Surgical Unit, Royal Children's Hospital, Flemington Rd, Melbourne VIC 3052, Australia (E-mail: ben.davies@mcri.edu.au).

J Thorac Cardiovasc Surg 2007;133:254-5

$0022-5223 / \$ 32.00$

Copyright @ 2007 by The American Association for Thoracic Surgery doi:10.1016/j.jtcvs.2006.09.009

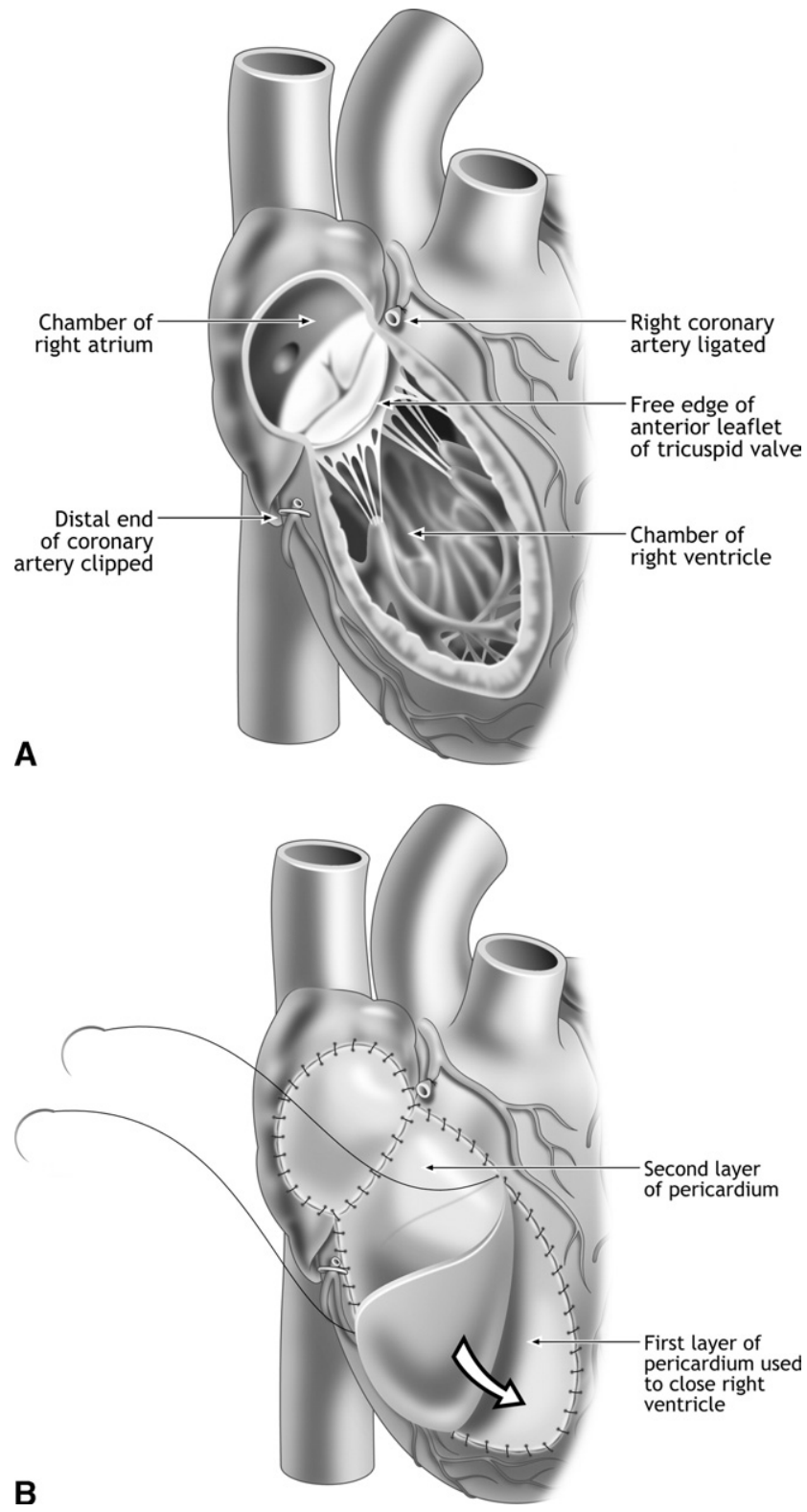

Figure 1. Diagrams illustrating extent of resection $(A)$ and subsequent reconstruction $(B)$.

PATIENT 2. A 4-month-old girl $(5.7 \mathrm{~kg})$ had intermittent runs of ventricular tachycardia since birth in association with an intracardiac mass. She was initially treated medically but referred for surgery when these episodes increased in duration and severity despite continuous intravenous amiodarone. Magnetic resonance 

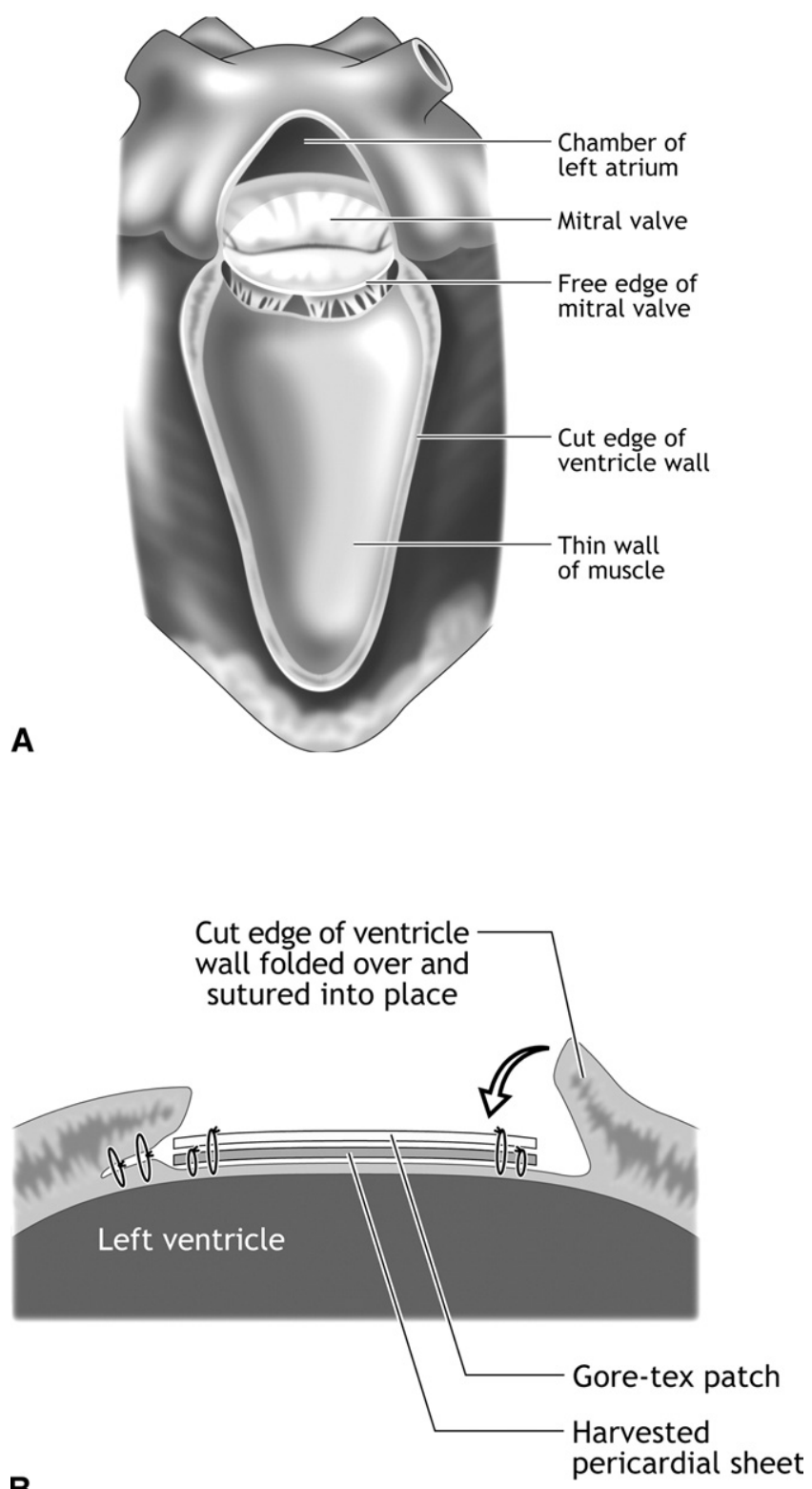

Figure 2. Diagrams demonstrating resection of left ventricular tumor (A) and multilayer reconstruction (B).

imaging and transesophageal echocardiography demonstrated a 6-cm mass within the inferoposterior left ventricular wall, extending from the apex across the AV groove into the left atrium involving the posterior aspect of the mitral annulus and circumflex artery. The remainder of the heart appeared normal with intermittent left-to-right shunting across a patent foramen ovale.

At surgery, the apex of the heart was displaced anteriorly, exposing the orange-sized mass. This was resected en bloc, removing ventricular tissue, circumflex artery, part of the posterior mitral annulus, and a portion of the left atrium. A paper-thin remnant of posterior ventricular wall approximately $40 \mathrm{~mm}$ wide was preserved between the basal portion of the ventricle and the apex. The resulting defect in the left atrium was reconstructed with a patch of pericardium (Figure E2), and the ventricular wall was reinforced by a single layer of autologous pericardium to which the posterior mitral leaflet had been sutured and reinforced with 0.6-mm thick expanded polytetrafluoroethylene membrane. Mattress sutures and fibrin glue were used to buttress and join the three layers (Figures 2; E3). Postoperative transesophageal echocardiography showed trivial mitral regurgitation, an akinetic posterior wall with good movement of the interventricular septum and anterior wall. The postoperative course was uneventful and the patient extubated 3 days later.

At follow-ups of 3.4 years and 8.4 months, respectively, both patients remain asymptomatic, in sinus rhythm, with trace AV valve regurgitation, good ventricular function, and without tumor recurrence.

\section{Discussion}

Primary cardiac tumors in children with medically refractory arrhythmias or hemodynamically significant obstruction unequivocally mandate complete resection. ${ }^{3}$ In extensive tumors, however, subtotal excision, orthotopic transplantation, or univentricular pathways have been advocated owing to concerns regarding the resection of key structures such as coronary arteries, conduction system, or the mere extent of the resection required and residual cardiac function. ${ }^{2,4}$

In both of our patients, the size and extent of the fibromas would put them in the realm of unresectable tumors. We postulated that from both the functional point of view, with the involved segment of the ventricular wall being de facto akinetic, and with the right or circumflex arteries already occluded from a vascular perspective, a virtual resection was already made. Providing that the surgery could restore the size of the cavity and sufficient rigidity to the ventricular wall to prevent dyskinetic motion, the complete excision of the tumors could be undertaken.

Complete resection of giant fibromas involving the AV groove can be accomplished. Reconstruction maintaining ventricular and valvular dimensions by simple means is easily achieved. The cardiac function at short- to medium-term follow-up is excellent, with resolution of symptoms.

\section{References}

1. Becker AE. Primary heart tumors in the pediatric age group: a review of salient pathologic features relevant for clinicians. Pediatr Cardiol. 2000;21:317-23.

2. Burke AP, Rosado-de-Christenson M, Templeton PA, Virmani R. Cardiac fibroma: clinicopathologic correlates and surgical treatment. J Thorac Cardiovasc Surg. 1994;108:862-70.

3. Stiller B, Hetzer R, Meyer R, Dittrich S, Pees C, Alexi-Meskishvili V, et al. Primary cardiac tumours: when is surgery necessary? Eur J Cardiothorac Surg. 2001;20:1002-6.

4. Aravot DJ, Banner NR, Madden B, Aranki S, Khaghani A, Fitzgerald $\mathrm{M}$, et al. Primary cardiac tumours-is there a place for cardiac transplantation? Eur J Cardiothorac Surg. 1989;3:521-4. 


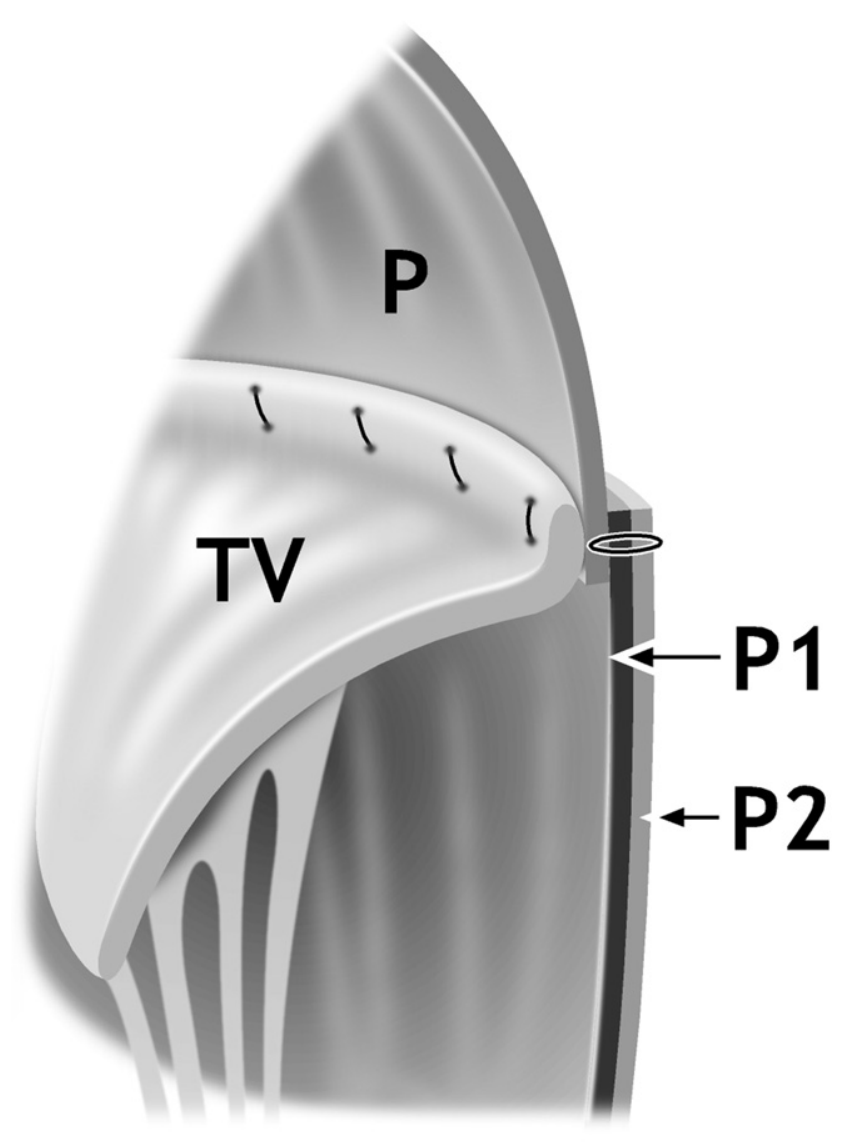

Figure E1. Patient 1: Restoration of atrioventricular junction and resuspension of TV annulus. P1 and P2, 1st and 2nd layers of glutaraldehyde-treated autologous pericardium. TV, Tricuspid valve.

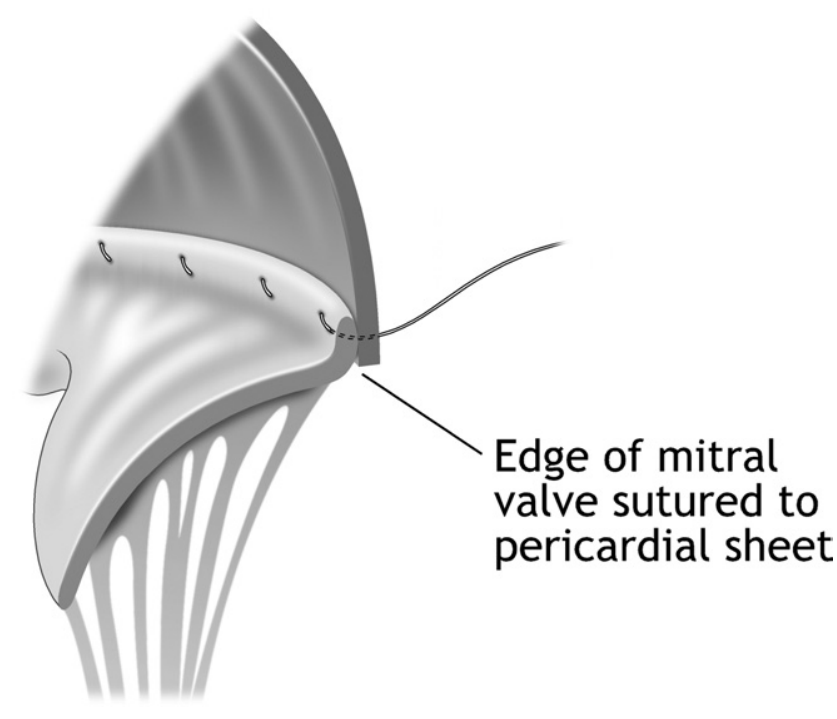

Figure E2. Patient 2: Attachment of posterior leaflet of mitral valve to pericardium.

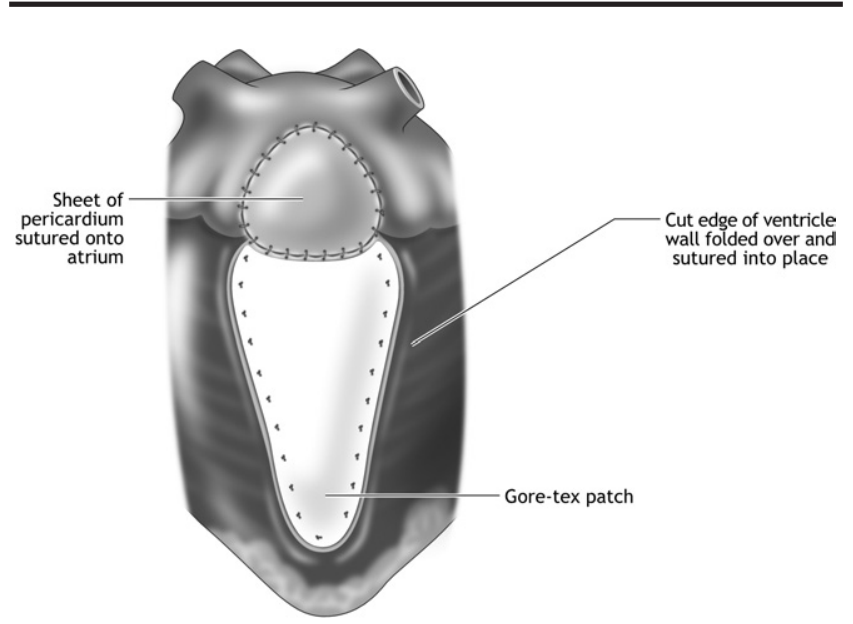

Figure E3. Patient 2: Completed reconstruction. 